\title{
Luminous binary supersoft X-ray sources: Optical colors and absolute magnitudes ${ }^{\star}$
}

\author{
V. Šimon ${ }^{\star \star}$ \\ Astronomical Institute, Academy of Sciences of the Czech Republic, 25165 Ondřejov, Czech Republic
}

Received 27 September 2002 / Accepted 15 April 2003

\begin{abstract}
An analysis of the color indices and absolute magnitudes $M_{V 0}$ of the "classical" supersoft X-ray binary sources (SSXBs), the V Sge-type stars (VSs), the symbiotic SSXBs, and the classical novae in the soft X-ray phase is presented. This approach can help comparing the properties and configuration of the reprocessing medium in the individual systems. The range of $M_{V 0}$ of the individual objects is very large, from $M_{V 0}=8.5$ to $M_{V 0}=-4$, most systems having $M_{V 0}<2$. The objects with the orbital period $P_{\text {orb }}<4$ days tend to possess brighter $M_{V 0}$ with the increasing $P_{\text {orb }}$. The same trend is apparent also for $M_{U 0}$. There is no systematic difference between the classical SSXBs and the VSs, as regards their $M_{V 0}$ and $M_{U 0}$. The soft X-ray phase of the novae V 1974 Cyg and V 382 Vel occurred at significantly brighter $M_{V 0}$ than those of most other SSXBs while $M_{V 0}$ of the recurrent nova U Sco was comparable to the SSXBs of the comparable $P_{\text {orb }}$. We showed that the luminosity of the reprocessing medium in the symbiotic SSXBs does not increase much with $P_{\text {orb }}$, when compared with the luminosity of the classical SSXBs and VSs; this speaks against large disks in these symbiotics. The classical SSXBs and the VSs have colors very similar each to other and form closed groups in the color-color diagrams. A Balmer jump occurs frequently in emission as inferred from the colors. The correlation between the bolometric luminosity, determined from the X-ray spectra and $M_{V 0}$ or $M_{U 0}$ of all kinds of the SSXBs is rather weak, but resolvable, and is more prominent for $M_{U 0}$. The implications for the reprocessing medium are discussed.
\end{abstract}

Key words. stars: binaries: close - stars: binaries: general - stars: circumstellar matter - stars: novae, cataclysmic variables

\section{Introduction}

The supersoft X-ray binaries (SSXBs) (e.g. CAL 83) are usually close systems, with their orbital periods $P_{\text {orb }}$ of the order of hours to a few days, in which the mass transfer onto a white dwarf (WD) occurs at a high rate $\left(\dot{m} \approx 10^{-7} M_{\odot} \mathrm{yr}^{-1}\right)$. This allows a steady-state hydrogen burning on the surface of the WD (van den Heuvel et al. 1992). This hydrogen burning is the source of the soft X-ray radiation. The opti$\mathrm{cal} / \mathrm{UV}$ radiation originates mostly from the reprocessing of the $\mathrm{X}$-rays in the disk and the donor star (Popham \& Di Stefano 1996). The known "classical" SSXBs are mostly located in the Magellanic Clouds and in M31. The reason is that the soft $\mathrm{X}$-rays are easily absorbed in the interstellar medium in our Galaxy.

In addition to these "classical" SSXBs, there are several groups of objects that could be their close relatives.

The V Sge-type stars (VSs) are close binaries which display very similar properties in the optical as the "classical" SSXBs. The potential members, based mainly on the similarities in their optical spectra, were listed by Steiner \& Diaz (1998). V Sge

* Tables 2 and 3 are only available in electronic form at http://www . edpsciences.org

$\star \star$ e-mail: simon@asu.cas.cz itself was detected as an X-ray source (Eracleous et al. 1991; Hoard et al. 1996). Greiner \& van Teeseling (1998) revealed that V Sge is a luminous, very soft X-ray source during the optical low state, turning into a faint hard source in the optical high state. They interpreted this behaviour in terms of a variable amount of circumstellar matter.

Some symbiotic stars can be supersoft X-ray sources, too (e.g. AG Dra; Greiner et al. 1997). These stars usually contain a WD, accreting matter from its red giant companion via a strong stellar wind or Roche lobe overflow. A search in the ROSAT archive by Mürset et al. (1996a) enabled them to sort the symbiotic stars into three categories according to the hardness of their X-ray spectra. Group $\alpha$ corresponds to the supersoft X-ray spectrum and contains Ln358, Dra C-1, R Aqr and CD-43 14304 . Group $\beta$ with a harder spectrum was attributed to the colliding winds. The hardest spectra of group $\gamma$ were supposed to represent the mass accreting neutron stars instead of the WD.

Several peculiar symbiotic novae may be related to the supersoft X-ray sources, too (e.g. RR Tel).

Some classical and recurrent novae were detected as luminous supersoft X-ray sources during the late decline phase of their outbursts (e.g. V 1974 Cyg/Nova Cyg 1992, Balman et al. 1998; GQ Mus/Nova Mus 1983, Shanley et al. 1995; U Sco, 
Table 1. The list of parameters of the SSXBs. The types of objects, defined in Sect. 2, are listed in the column labeled Type (class. refers to the classical SSXB). The table further gives the distance $d$ (kiloparsecs), orbital period $P_{\text {orb }}$ (days), maximum and minimum apparent visual magnitude $V_{\max }$ and $V_{\min }$, respectively, the reddening $E(B-V)$ and the interstellar absorption $A_{\mathrm{V}} . M_{V 0 \max }$ and $M_{V 0 \min }$ refer to the maximum and minimum dereddened absolute visual magnitude, respectively. $L_{\mathrm{bolBB}}$ and $L_{\mathrm{bolWD}}$ represent the bolometric luminosities in erg $\mathrm{s}^{-1}$, determined from the X-ray spectra by the black-body and WD atmosphere fits, respectively.

\begin{tabular}{|c|c|c|c|c|c|c|c|c|c|c|c|}
\hline Object & Type & $d(\mathrm{kpc})$ & $P_{\text {orb }}$ (days) & $V_{\max }$ & $V_{\min }$ & $E(B-V)$ & $A_{V}$ & $M_{V 0 \max }$ & $M_{V 0 \min }$ & $L_{\text {bolBB }}$ & $L_{\text {bolWD }}$ \\
\hline \multicolumn{12}{|l|}{ Galaxy } \\
\hline QR And & class. & 2 & 0.6605 & 11.5 & 12.65 & 0.10 & 0.31 & -0.32 & 0.83 & & $4 \times 10^{36}$ \\
\hline MR Vel & class. & 1.5 & 4.0288 & 17.1 & 17.3 & 2 & 6.2 & 0.02 & 0.22 & & $4 \times 10^{35}$ \\
\hline V Sge & VS & 4 & 0.514 & 10.3 & 12.4 & 0.33 & 1.02 & -3.73 & -1.63 & & $\leq 1.6 \times 10^{37}$ \\
\hline V617 Sgr & VS & & 0.2071 & & 14.8 & 0.2 & 0.62 & & & & \\
\hline WX Cen & VS & 1.4 & 0.4167 & & 13.7 & 0.4 & 1.24 & & 1.73 & & \\
\hline HD 45166 & VS & 1.26 & 0.357 & & 9.88 & 0.15 & 0.46 & & -1.08 & & \\
\hline V1974 Cyg & nova & 1.77 & 0.0812 & & 9.5 & 0.32 & 0.99 & & -2.73 & & $7.9 \times 10^{37}$ \\
\hline GQ Mus & nova & 4.8 & 0.0594 & 17.5 & 18.0 & 0.45 & 1.39 & 2.70 & 3.20 & $\approx 10^{38}$ & $\approx 10^{37}$ \\
\hline V382 Vel & nova & 1.7 & 0.14615 & & 9.05 & 0.1 & 0.31 & & -2.41 & $\approx 10^{38}$ & \\
\hline U Sco & nova & 6 & 1.23056 & & 13.3 & 0.2 & 0.62 & & -1.21 & $2.3 \times 10^{36}$ & $2.4 \times 10^{37}$ \\
\hline RR Tel & symb. & 2.6 & & & 10.84 & 0.1 & 0.31 & & -1.54 & & $1.3 \times 10^{37}$ \\
\hline AG Dra & symb. & 1.7 & 550 & 8.46 & 9.85 & 0.06 & 0.19 & -2.88 & -1.49 & $4.4 \times 10^{36}$ & \\
\hline 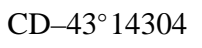 & symb. & 1.5 & 1448 & 12.14 & 12.53 & $\sim 0.2$ & $\sim 0.6$ & 0.66 & 1.05 & & \\
\hline R Aqr & symb. & 0.25 & $44 \mathrm{yr} ?$ & 7.5 & 12.0 & 0.0 & 0.0 & 0.5 & 5.0 & & \\
\hline V751 Cyg & $\mathrm{CV}$ & 0.5 & 0.1445 & 13.6 & 17.8 & 0.25 & 0.77 & 4.34 & 8.54 & $\leq 6.5 \times 10^{36}$ & \\
\hline \multicolumn{12}{|l|}{ LMC } \\
\hline J0513 & class. & 47.3 & 0.76278 & 16.4 & 17.55 & 0.1 & 0.31 & -2.28 & -1.13 & & $7 \times 10^{37}$ \\
\hline J0439 & class.? & 47.3 & 0.1404 & & 21.74 & 0.1 & 0.31 & & 3.06 & & $9 \times 10^{37}$ \\
\hline J0537 & class. & 47.3 & 0.1458 & & 19.66 & 0.1 & 0.31 & & 0.98 & $8.5 \times 10^{36}$ & \\
\hline CAL 83 & class. & 47.3 & 1.0417 & 16.9 & 17.5 & 0.1 & 0.31 & -1.78 & -1.18 & $5.8 \times 10^{37}$ & $\geq 2 \times 10^{37}$ \\
\hline CAL 87 & class. & 47.3 & 0.4425 & & 18.9 & 0.05 & 0.15 & & 0.38 & & $5 \times 10^{36}$ \\
\hline \multicolumn{12}{|l|}{ SMC } \\
\hline 1E0035 & class. & 57.5 & 0.17193 & & 20.3 & 0.06 & 0.19 & & 1.31 & $3 \times 10^{37}$ & $5 \times 10^{36}$ \\
\hline J0048 & symb. & 57.5 & & & 15.0 & 0.05 & 0.15 & & -3.95 & & $4.8 \times 10^{37}$ \\
\hline Ln358 & symb. & 57.5 & & & 15.2 & 0.08 & 0.25 & & -3.85 & & \\
\hline \multicolumn{12}{|c|}{ Draco dwarf galaxy $C$} \\
\hline Draco $\mathrm{C}-1$ & symb. & 76 & & 16.8 & 17.14 & 0.03 & 0.1 & -2.70 & -2.36 & & \\
\hline
\end{tabular}

Kahabka et al. 1999b). The radius of the H-burning WD already shrinks during this phase and its temperature is sufficient to emit soft X-rays and to irradiate the already re-established accretion disk.

It appears interesting and important to compare the optical properties of the individual categories of the supersoft X-ray sources, particularly their luminosities and color indices. This may help comparing the properties and configuration of the reprocessing medium in the individual systems.

A preliminary version of this analysis was presented by Šimon (2002).

\section{Sources of the data}

This analysis considers several types of SSXBs: the classical SSXBs, VSs, classical and recurrent novae with the soft X-ray emission, and the symbiotic SSXBs. One cataclysmic variable, V $751 \mathrm{Cyg}$, that displayed the soft X-ray emission during its optical low state, is also included. The electronic catalog of supersoft X-ray sources available at URL http://www.mpe.mpg.de/ jcg/sss/ssscat.html and maintained by J. Greiner served as the first guide in the search for the data. The list of the objects with their adopted and derived parameters is given in Table 1. The summary of the literature used can be found in Table 2. The colors of the objects are summarized in Table 3. Tables 2 and 3 are available only in electronic form.

\section{Data analysis}

At least one color index could be determined for each object, listed in Table 1. The index was determined from the 
closely spaced observations, obtained in a single night. In some cases, the mean indices were determined from the folded orbital curves, composed from observations obtained during several nights. Typical errors of the color indices are of the order of a few hundredths of mag. The indices were dereddened using $E(B-V)$.

The absolute dereddened visual magnitude $M_{V 0}$ was calculated from the distance $d$ and $E(B-V)$. The distances corresponded to the distances of LMC, SMC and Draco dwarf galaxy for the members of these galaxies. The new values of the distances $47.3 \mathrm{kpc}$ and $57.5 \mathrm{kpc}$ were adopted for LMC (Gould 1995) and SMC (van den Bergh 1989), respectively. The methods and procedures used for the determination of the distances of the objects in our Galaxy are described in the original references, listed in Table 2. The distance was often determined from the strength of the interstellar absorption feature in the UV region near $\lambda=220 \mathrm{~nm}$. In the case of some symbiotics, the distance was determined from the luminosity of the cool giant, appropriate to its spectral type. In most cases, also $E(B-V)$ was determined from the strength of the feature near $\lambda=220 \mathrm{~nm}$ (see Table 2 for references).

\subsection{Absolute magnitudes}

In principle, $M_{V 0}$ of the SSXBs can depend on their orbital period $P_{\text {orb }}$ because the radius of the Roche lobe of the WD (and hence of the disk) depends on $P_{\text {orb }} . V_{\max }$ and $V_{\text {min }}$, listed in Table 1, refer to the extreme apparent visual magnitudes of the long-term activity at which a given object has ever been seen. For the novae and RR Tel, just the magnitudes corresponding to the phase of the soft X-ray emission (or close to it) are listed. $M_{V 0}$ 's were then plotted versus $P_{\text {orb }}$ in Fig. 1a. $P_{\text {orb }}$ 's of RR Tel, Dra C-1, Ln358 and J0048 are unknown but are supposed to be of the order of hundreds of days or even several years. $M_{V 0}$ 's of the novalike variables and nova remnants, taken from Warner (1995), are plotted for comparison. The $U$ band is more suitable for the assessment of the light contribution of the hot component of the symbiotic SSXBs (Fig. 1b).

The reprocessing of the X-rays from the H-burning WD in the disk and the companion is believed to be an important source of the UV and optical light. It is therefore meaningful to investigate the relation between $M_{V 0}$ and the bolometric luminosity $L_{\mathrm{bol}}$. In the case of the SSXBs, $L_{\mathrm{bol}}$ is determined from the fit to their X-ray spectra. Both the black-body fits and fits by the WD model atmosphere are used by the individual authors (Table 1). The resulting diagrams are shown in Fig. 2. Both $M_{V 0}$ and $M_{U 0}$ are used because in principle the reprocessing may differ in the optical and near UV region. $M_{V 0}$ and $M_{U 0}$ in Fig. 2 more or less correspond to the level of the optical activity for which $L_{\text {bol }}$ was measured. Notice that the scatter is large, particularly in Fig. 2a.

\subsection{Color indices}

Both the observed and dereddened color indices were plotted in the color-color diagrams (Fig. 3) - this arrangement was chosen because the reddening may not be quite reliable for some
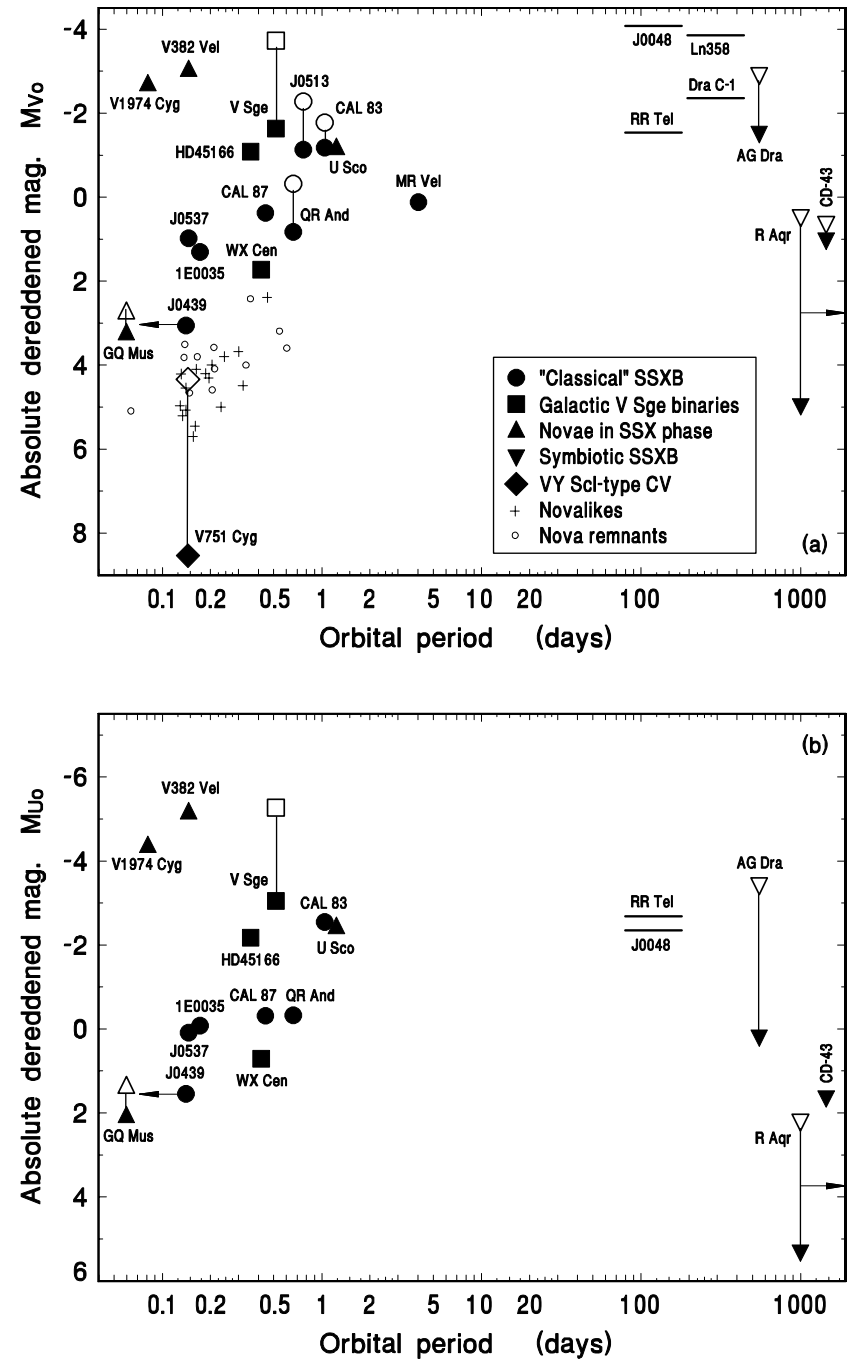

Fig. 1. The absolute dereddened visual magnitudes $M_{V 0}$ a) and ultraviolet magnitudes $M_{U 0}$ b) plotted versus the orbital periods $P_{\text {orb }}$. The individual categories of objects are resolved. The filled symbols denote the normal level of $M_{V 0}$ or a low state while the empty symbols refer to the high state. The lengths of $P_{\text {orb }}$ of the symbiotics RR Tel, Dra C-1, Ln358 and J0048 are unknown. A possible $P_{\text {orb }}$ of R Aqr is about 44 years (off scale). $M_{V 0}$ of the novalike variables and nova remnants are plotted for comparison. See Sect. 3.1 for details.

objects. The colors of the main-sequence stars, giants, and supergiants, according to Johnson (1966), are included for comparison. The colors of the classical novae V 1974 Cyg and V 382 Vel were determined, but not included in the diagrams because they displayed a significant scatter, due to the large strengths of the emission lines from their ejecta.

$U-B$ vs. $B-V$ diagram (Fig. 3a): most displayed objects form a closed group, except for the symbiotic SSXBs. Some objects even lie above the curve of black bodies. This fact is not affected by the possible uncertainties in $E(B-V)$ (Fig. 3b). Notice also that the region of the short-period systems ( $P_{\text {orb }} \leq 4$ days) can be separated from that of the long-period ones according to the $(B-V)_{0}$ index.

$(U-B)_{0}$ vs. $(B-V)_{0}$ diagram (the classical SSXBs and VSs only) (Fig. 4): the mean colors are plotted. The individual 

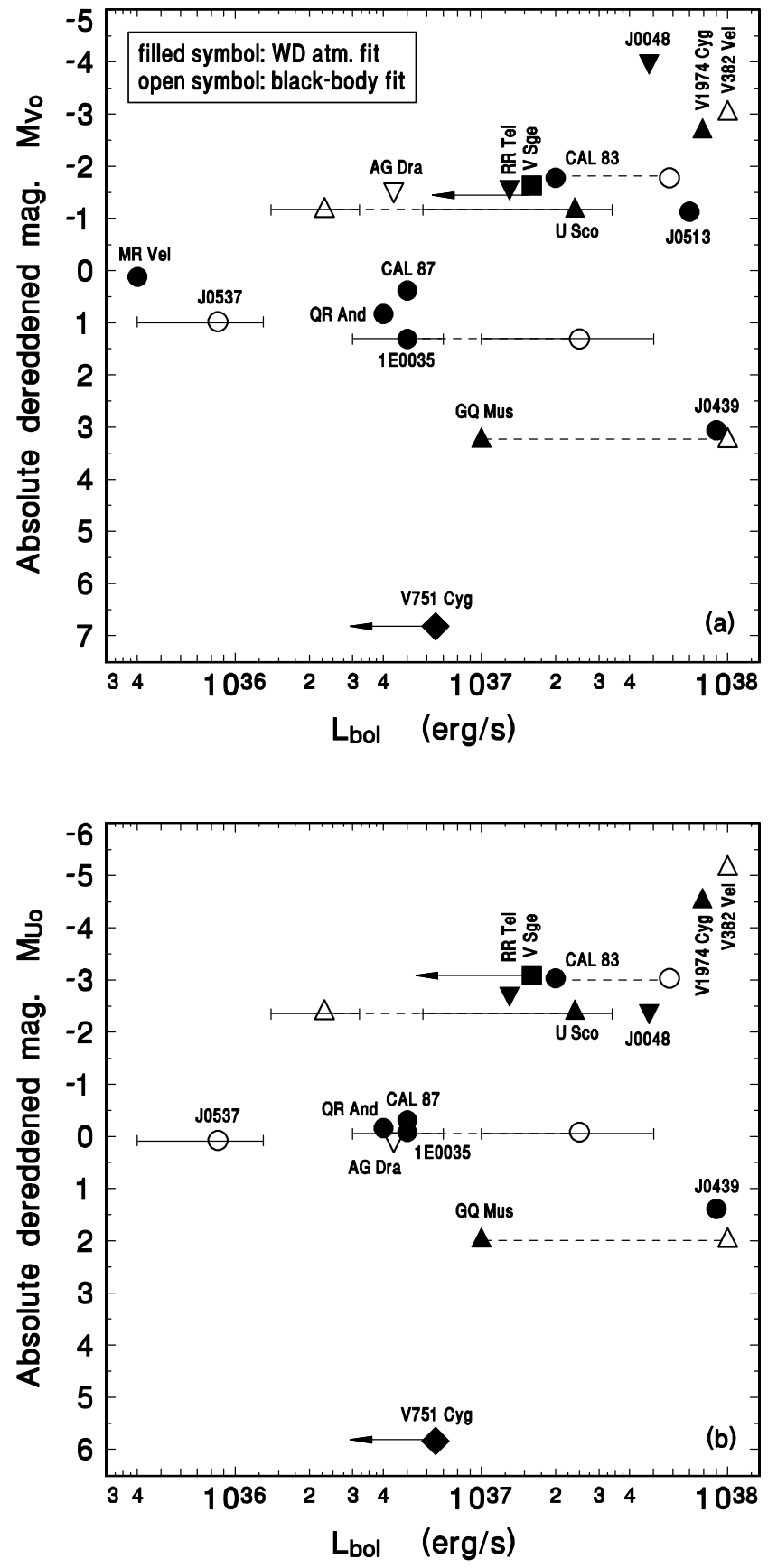

Fig. 2. $M_{V 0}$ a) and $M_{U 0}$ b) plotted versus the bolometric luminosity $L_{\mathrm{bol}}$, determined from the X-ray spectra. The individual categories of objects as in Fig. 1 are resolved but here the empty and the filled symbols denote the black-body and WD atmosphere fits, respectively. For convenience, the dashed line connects the values of $L_{\mathrm{bol}}$ of a given object, determined by the individual methods. The absence of the error bar means that none was given in the reference, listed in Table 2. $L_{\mathrm{bol}}$ of V Sge is subject to a large uncertainty; the symbol represents the upper limit. See Sects. 3.1 and 3.2.1 for details.

objects form a group elongated along the diagonal. The size of the symbol reflects the mean absolute magnitude of the object in $V$. There is some tendency for the $\mathrm{V}$ Sge stars to possess more negative $(B-V)_{0}$ 's at more positive $(U-B)_{0}$ 's.
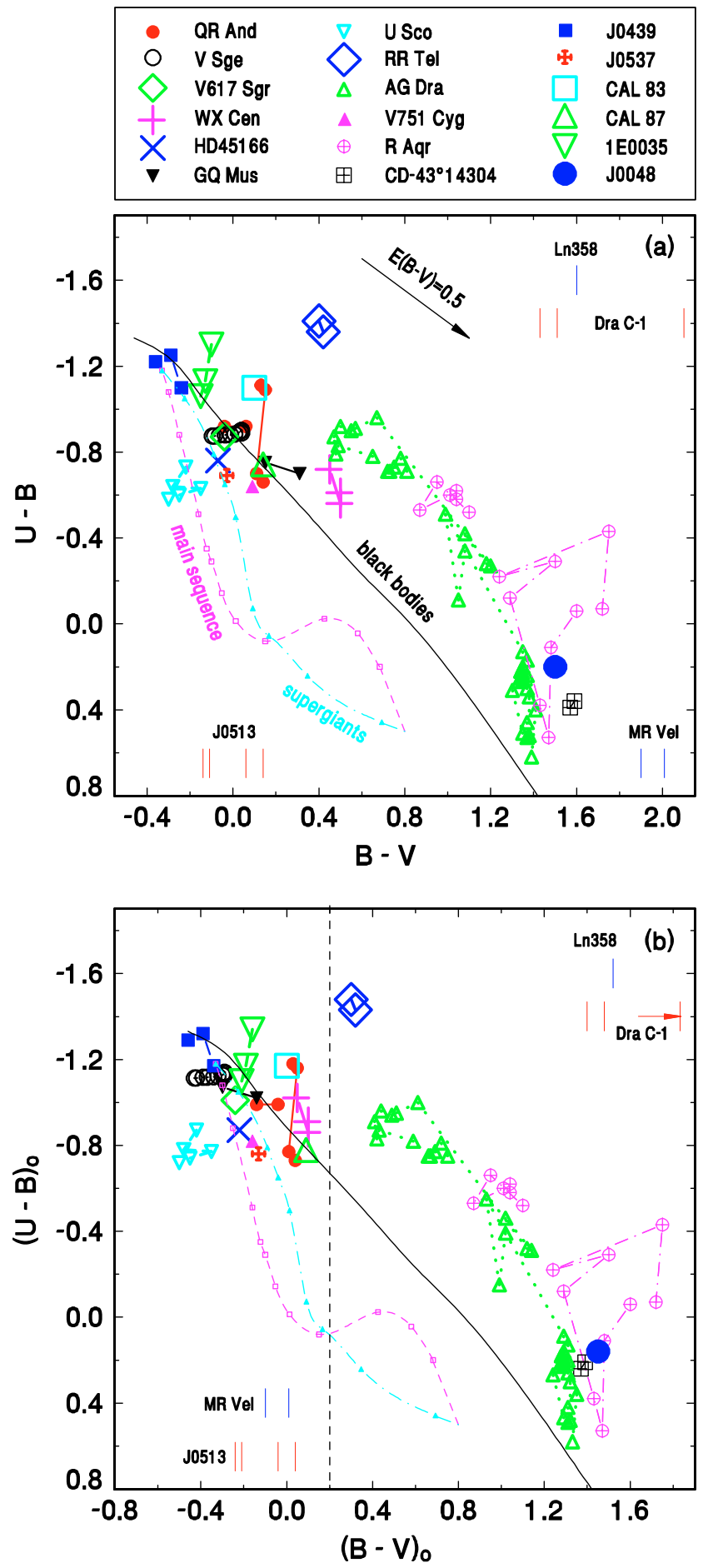

Fig. 3. $U-B$ vs. $B-V$ diagram a) and $(U-B)_{0}$ vs. $(B-V)_{0}$ diagram b). All available measurements of the sources are shown. Notice that some objects lie above the curve of black bodies; this fact is not affected by the possible errors in $E(B-V)$. The vertical dashed line in b) separates the region of the short-period systems ( $P_{\text {orb }} \leq 4$ days) from the long-period ones. The short vertical lines denote the $B-V$ indices of the objects for which no $U-B$ colors are available. See Sect.3.2 for details. (This figure is available in color in electronic form.)

$B-V$ vs. $V-R$ and $V-R$ vs. $R-I$ diagrams: The clustering of the colors of the short-period systems is apparent, similar to Fig. 3. The separation of the colors of the short-period and symbiotic systems is large, the latter being close to the red giants. 


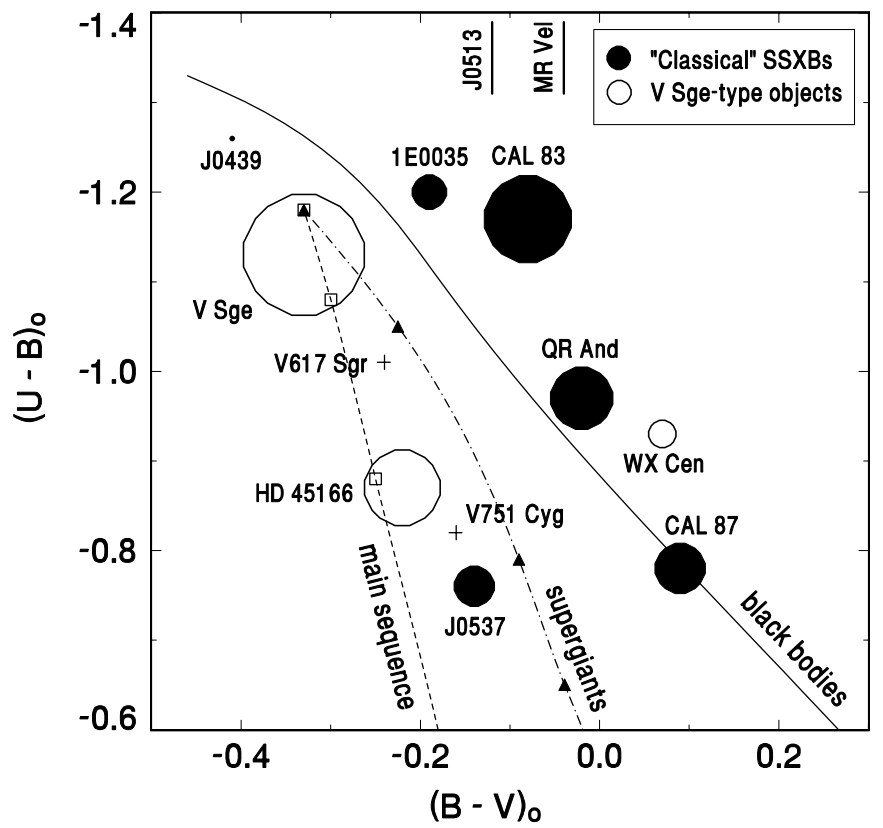

Fig. 4. $(U-B)_{0}$ vs. $(B-V)_{0}$ diagram just for the classical SSXBs and VSs. The mean colors of each object are plotted. The size of the symbol is proportional to the mean $M_{V 0}$ of a given object. $M_{V 0}$ of V 617 Sgr is unknown and $M_{V 0}$ of V 751 Cyg in the low state falls off scale. Just $(B-V)_{0}$ is available for J0513 and MR Vel. See Sect. 3.2 for details.

$(B-V)_{0}$ vs. $M_{V 0}$ and $(U-B)_{0}$ vs. $M_{V 0}$ diagrams: no clear correlation is apparent when the individual systems are compared. AG Dra displays a long track toward smaller index during the rise of its brightness. A very weak correlation may appear only when $M_{U 0}$ is used; the symbiotics tend to be fainter and redder than the classical SSXBs and VSs.

$P_{\text {orb }}$ vs. $(B-V)_{0}$ and $P_{\text {orb }}$ vs. $(U-B)_{0}$ diagrams: it can be expected that the systems with longer $P_{\text {orb }}$ will possess larger disks (e.g. Paczynski 1977). Larger disks can be cooler (redder) in their outer parts than smaller disks, provided that $\dot{m}$ remains the same. However, it was found that neither $(B-V)_{0}$ nor $(U-B)_{0}$ display any clear dependence on $P_{\text {orb }}$ for the systems with $P_{\text {orb }}<4$ days.

\subsubsection{Comments on some systems}

The observed values used for some systems in this analysis need comments.

The mean colors, based on the fits to the color vs. $\operatorname{mag}(V)$ were used for V Sge (Šimon et al. 2001). Greiner \& van Teeseling (1998) admitted a large range of the possible parameters of the fit to its X-ray spectrum. $L_{\text {bolwD }}$ may be as large as $10^{36} \mathrm{erg} \mathrm{s}^{-1}$ for $d=1 \mathrm{kpc}$. Their $L_{\text {bolwD }}$ was rescaled to $d=4 \mathrm{kpc}$ in Table 1 and Fig. 2 . The distance of $4 \mathrm{kpc}$ should be preferred because the UV data are much better fitted by the non-standard extinction curve (Smak et al. 2001).

The color indices of $\mathrm{QR}$ And represent just the low/intermediate state of its activity because the rare high states (outbursts?) were observed just on the archival plates (Greiner \& Wenzel 1995).
Just the colors corresponding to the phase of the detected soft X-ray emission, and not to the complete explosion, were used for U Sco and RR Tel. The colors just preceding this phase, but no colors exactly corresponding to it, were available for U Sco; these indices change just a little during the outburst for $t-T_{\max }>5$ days (Table 3 ).

The available optical colors and magnitudes of Ln358, Dra C-1, R Aqr, and CD-43 14304 are not simultaneous to the X-ray observations. Among these, only R Aqr appears to display large changes of its optical brightness, mostly due to its Mira component. It therefore appears acceptable to use the non-simultaneous data in these cases.

The colors of CD-43 14304 in 1987 and 1991 refer to the continuum only, with the contribution of the emission lines subtracted.

The color indices of R Aqr consist of two sets. The points of each set are connected by the lines to show the temporal evolution. The latter set covers one full pulsation cycle of the Mira-type component.

The colors of AG Dra capture a part of a quiescent interval and subsequent two outbursts.

The indices $V-R$ and $R-I$ of CAL 87 were determined from the out-eclipse intervals of the curves folded with $P_{\text {orb }}$.

\section{Discussion}

Here we present a comprehensive analysis of the optical colors and absolute magnitudes of the binaries with the luminous soft $\mathrm{X}$-ray emission and their close relatives.

\subsection{Absolute magnitudes of the short-period systems}

The range of $M_{V 0}$ 's of the individual objects, emitting the soft $\mathrm{X}$-rays, is shown to be very large, from $M_{V 0}=8.5$ (V 751 Cyg) to $M_{V 0}=-4$ (J0048). The largest part of the systems with $P_{\text {orb }} \leq 4$ days displays $M_{V 0}<2$. The classical SSXBs and the VSs tend to display brighter $M_{V 0}$ with the increasing $P_{\text {orb }}$ and there are no systematic differences between these two groups in the $P_{\text {orb }}-M_{V 0}$ plot; the same is true also for $M_{U 0}$. All this is in accordance with the hypothesis outlined above - the longer $P_{\text {orb }}$ allows a larger disk, on which the soft X-rays can be reprocessed.

For a given $P_{\text {orb }}$, the lower limit of $M_{V 0}$ of the classical SSXBs is set by $\dot{m}$ necessary to maintain a steady-state H-burning while the upper limit can be expected to be set by the maximum dimensions of the disk (although a circumbinary envelope contributes in the high state of V Sge, Herbig et al. 1965 and maybe also in QR And Kuduz et al. 2002). The same trend in the $P_{\text {orb }}-M_{V 0}$ diagram is observed also for the novalike variables and the nova remnants. Their $M_{V 0}$ 's are systematically fainter than those of the SSXBs but some objects almost overlap with them. It therefore appears that there may not be quite a sharp boundary and a large gap between the classical SSXBs+VSs and the brightest novalikes and nova remnants. Several possible causes of the higher $\dot{m}$ of the classical SSXBs and VSs can be assumed. A high mass ratio $q=M_{\text {donor }} / M_{\mathrm{WD}} \geq 1$ will lead to a thermally unstable mass transfer (van den Heuvel et al. 1992). Another possibility 
for the SSXBs with $q<1$ can be an induced wind of the donor (van Teeseling \& King 1998; Knigge et al. 2000). The role of the latter scenario may be more important than thought previously because QR And which had been supposed to have $q>1$ was proven to have $q<1$ (McGrath et al. 2001).

The currently available observations show that the high/low states (or some kind of outburst) occur just for the classical SSXBs and VSs with $P_{\text {orb }}=0.5-1.1$ days. Nevertheless, the amplitude in such a system is still smaller than the total range of $M_{V 0}$ of the objects in Fig. 1 .

$M_{V 0}$ of the recurrent nova U Sco was comparable to the SSXBs of similar $P_{\text {orb }}$, in variance with the classical novae V 1974 Cyg and V 382 Vel. This can be explained by the mass of the ejecta in U Sco by almost two orders of magnitude lower (3-9 $\times 10^{-7} M_{\odot}$, Iijima 2001; Woodward et al. 1997; Della Valle et al. 2002). $M_{V 0}$ and the colors of U Sco just preceding its soft X-ray phase therefore represent mostly the optically thick wind from the WD and the disk which should re-establish in that $t-T_{\max }$ (see the model by Hachisu et al. 2002). The decline of the outburst of U Sco went through the soft X-ray phase at $M_{V 0}$ and $M_{U 0}$ comparable to the classical SSXBs and VSs of a similar $P_{\text {orb }}$ (Fig. 1); this implies similar disk parameters.

The soft X-ray emission of V 751 Cyg is detected just in its optical low state (Greiner et al. 1999). Patterson et al. (2001) argued that this emission is too high and should lead to a prominent reflection effect on the donor. We note that this effect could be precluded by the disk contribution because V 751 Cyg was at about $16 \operatorname{mag}(V)$, almost $2 \mathrm{mag}$ above the deepest level. V 751 Cyg may help to explain why most Galactic VSs are not luminous X-ray sources. Maybe only when the disk is almost missing the soft X-rays can escape. The high metallicity in comparison with the objects in LMC and SMC can play a role (Rauch 1996).

\subsection{The optical to bolometric luminosity ratios}

We have shown that the relation between $M_{V 0}$ and $L_{\text {bol }}$ that can give us important information about the reprocessing of the soft $\mathrm{X}$-rays displays a large scatter (Fig. 2). Several causes, like the inclination of the disk and the intrinsic absorption of the soft $\mathrm{X}$-rays in the outflows, can give rise to this scatter. The reason is that in the case of the SSXBs $L_{\text {bol }}$ is determined from the spectral fit in the soft X-ray region because the flux is heavily absorbed in the EUV passband. $L_{\text {bol }}$ then strongly depends on the temperature and absorption, which are strongly correlated. The uncertainties involved may be demonstrated on the case of V Sge and MR Vel. The interstellar column density towards V Sge is $N_{\mathrm{H}} \approx 1.6 \times 10^{21} \mathrm{~cm}^{-2}$ (Smak et al. 2001) but the total $N_{\mathrm{H}}$, including the intrinsic extinction, may be as high as $4 \times 10^{22} \mathrm{~cm}^{-2}$ (model-dependent) (Greiner $\&$ van Teeseling 1998). This is comparable to the high $N_{\mathrm{H}} \approx 1.3 \times 10^{22} \mathrm{~cm}^{-2}$ of MR Vel (Motch et al. 2002). Greiner \& van Teeseling (1998) also admitted that we may see just a small portion of the surface of the WD in V Sge and that the fit of the X-ray spectrum is poorly constrained. We note that V Sge possesses a considerably higher orbital inclination and much stronger activity than MR Vel, which can contribute to the obscuration of its WD by the circumstellar matter. Also the complicated profile of the X-ray spectrum of MR Vel which cannot be fitted by the WD model atmosphere in its full complexity (Motch et al. 2002) may contribute to its low inferred $L_{\text {bol }}$.

Also a strong anticorrelation between the optical and X-ray intensity of some systems can affect the scatter (V Sge: Greiner \& van Teeseling 1998; AG Dra: Greiner et al. 1997; J0513: Reinsch et al. 2000). The contribution of the donor can affect the scatter, too (e.g. AG Dra in $V$ ). The outstanding position of J0439 can be due to its possible ultra-short $P_{\text {orb }}$ (van Teeseling et al. 1997) and hence to a lack of extensive reprocessing medium. Nevertheless, some trends can be resolved in Fig. 2 if we give a higher weight to the WD atmosphere fits than to the black-body fits. Then, the systems with a higher $L_{\text {bol }}$ often display a brighter $M_{V 0}$, and particularly $M_{U 0}$. A better correlation of $L_{\mathrm{bol}}$ with $M_{U 0}$ than with $M_{V 0}$ can suggest that the reprocessing is more complicated in $V$ than in $U$. In summary, the reprocessing of the soft X-rays largely differs from system to system. It would therefore be premature to reject most VSs from the potential SSXBs. Yet undetected SSXBs can be hidden among them and could be revealed according to their highexcitation emission lines and blue colors in the optical.

\subsection{The colors of the short-period systems}

We have found that the short-period systems ( $P_{\text {orb }} \leq 4$ days $)$ form closed groups in the color-color diagrams, spanning over the $U$ to the $I$ passband, despite their largely divergent $M_{V 0}$ and $P_{\text {orb }}$. There are no significant dependences between $M_{V 0}$ and $(B-V)_{0}$ or $(U-B)_{0}$. The slopes of their spectra therefore display large similarities in this spectral region, regardless the optical luminosity. In addition, we have found no dependence of color on $P_{\text {orb }}$. All this can be explained in terms of a flatter radial temperature profile of an irradiated disk in comparison with a standard disk, radiating purely due to the viscous processes. The lack of the dependence of color on $P_{\text {orb }}$ of the SSXBs also suggests a large difference between the luminosity of the disk and the donor star, causing thus that the donor will not affect the colors very much by its intrinsic luminosity, perhaps only by the reprocessing. On the contrary, the "ordinary" CVs above the period gap do show a reddening trend with the longer $P_{\text {orb }}$ (Hack \& la Dous 1993). The colors of most shortperiod SSXBs are comparable with those of the bulk of the old novae (Szkody 1994) in the $B$ to $R$ region. It is likely that in the old novae an irradiation of the disk by the still hot WD occurs at some extent, too. The similar colors then may suggest that also the temperatures of the outer parts of the disks in these two kinds of objects are comparable and that the irradiation of these parts is not extremely high in the SSXBs.

Smaller differences in the positions of the short-period systems in the color-color diagrams are apparent. The $(U-B)_{0}$ vs. $(B-V)_{0}$ diagram displays a diagonal elongation, with the color variations of the individual objects smaller than the total range of indices of the group. This suggests that the properties of the reprocessing medium are characteristic for a given binary and undergo just relatively small changes during various states of activity and due to the orbital motion. The differences 
in the flaring disk and the donor star in the individual systems can also play a role. Notice for example the difference between $(U-B)_{0}$ of $1 \mathrm{E} 0035$ and J0537 at very similar $(B-V)_{0}$ 's (Fig. 4). Both systems have quite similar $M_{V 0}, P_{\text {orb }}$ and $L_{\text {bol }}$. The flaring disk and the donor are more prominent at longer wavelengths, therefore the systems with a larger contribution of these components would be expected to be redder. The position of some systems with $P_{\text {orb }} \leq 4$ days above the curve of radiating black bodies in Fig. $3 \mathrm{~b}$ also suggests a substantial line contribution (e.g. Balmer jump affected by emission). The colors of U Sco around its soft X-ray phase are quite similar to the classical SSXBs and VSs, in accordance with the very low mass of its ejecta (see above).

The $(U-B)_{0}$ and $(B-V)_{0}$ indices of J0439 are the most negative of the objects plotted in Fig. 4. At the same time, J0439 has the faintest $M_{V 0}$ of the classical SSXBs and VSs (Figs. 1 and 4). These facts speak in favour of a hot, but spatially small reprocessing medium (disk?). This supports the suggestion by van Teeseling et al. (1997) that J0439 is an extremely hot WD or a double-degenerate SSXB, with the real $P_{\text {orb }}$ shorter than 0.1404 days, previously determined by Schmidtke \& Cowley (1994). Also the high $L_{\text {bol }}$ and faint $M_{V 0}$, discussed above, support these conclusions.

\subsection{The symbiotic SSXBS}

$M_{V 0}$ of the symbiotic SSXBs are comparable to or just slightly brighter than those of the brightest classical SSXBs and VSs with a much shorter $P_{\text {orb }} . M_{U 0}$ even shows that the hot components of these symbiotics are fainter than the brightest classical SSXBs and VSs. The luminosity of the medium in these symbiotics (ionized envelope, wind, accretion disk?) in which the reprocessing of the soft X-rays occurs therefore does not increase much with $P_{\text {orb }}$. The problem of the presence or absence of the accretion disks in the symbiotics is far from being solved (e.g. Hack \& la Dous 1993). Our comparison of $M_{V 0}$ 's of the classical SSXBs and the symbiotic SSXBs speaks in favour of a diluted medium in the latter and against their large disks because their irradiation by the hot WDs would lead to $M_{U 0}$ and $M_{V 0}$ much brighter than observed and brighter than in the classical SSXBs. The minimum state of the luminosity of R Aqr is even comparable to that of V $751 \mathrm{Cyg}$ in the low state. It is also interesting to note that while the soft X-ray emission ceased during the outburst of AG Dra, it is usually still observable in the classical SSXBs at this $M_{V 0}$. The expansion of the radius of the WD during the outburst of AG Dra (Greiner et al. 1997) can therefore offer another effect which can be responsible for the missing soft X-ray emission of many VSs.

The positions of the symbiotic SSXBs in the $(U-B)_{0}$ vs. $(B-V)_{0}$ diagram (Fig. 3) are mostly different from those of the remaining objects of this study. They lie on a long diagonal track above the line of the radiating black bodies, which implies mainly the Balmer jump in emission. The variable prominence of the hot component between the quiescence and the outburst (or during the pulsation cycle) also plays a role. The $(U-B)_{0}$ index, and at some extent also $(B-V)_{0}$ of RR Tel and AG Dra in outburst are not very different from those of the short-period systems (Fig. 3b). This may suggests some similarity of the slope of their blue and near UV spectra but one has to be cautious - the spectrum of the symbiotic SSXBs in this region is usually dominated by the recombination continuum but the WR-type wind from the hot component may contribute. Some models even showed that a structure similar to a disk may be formed even in the wind accretion (Theuns \& Jorissen 1994). If really such disks are present in the symbiotic SSXBs, then their dimensions are not much larger than in the classical SSXBs because of the reasons given in the preceding paragraph.

There appear to be two groups of the symbiotic SSXBs: symbiotic novae (RR Tel and probably J0048) and quiescent symbiotics (AG Dra and most others). The symbiotic novae have a strong soft X-ray emission during their optical outburst. On the contrary, AG Dra displays this emission just in its quiescent state (Greiner et al. 1997) when its $M_{U 0}$ is comparable to that of the optically faint classical SSXBs. CD-43 ${ }^{\circ} 14304$, Dra $\mathrm{C}-1$ and $\mathrm{R}$ Aqr display even fainter $M_{U 0}$. Two factors may contribute to the detectability of the soft X-ray emission in these systems - low metallicity (e.g. CD-43¹4304, AG Dra: Schmid \& Nussbaumer 1993; Dra C-1: Munari \& Buson 1994) and/or a relatively low optical luminosity. The low metallicity implies a low absorption of the soft X-rays and their easy escape from the system. The low optical luminosity implies a low amount of the circumstellar matter and hence a low absorption of the soft X-rays, but also less matter to reprocess on. These symbiotics may therefore be the systems that underwent thermonuclear outbursts in the past (e.g. decades ago) and $\mathrm{H}$-burning of the remaining, non-ejected matter on the surface of their WDs still continues (this was suggested for AG Dra by Greiner et al. 1997). In addition, their mass transfer appears to be low, as suggested not only from their low optical/ near UV luminosity, but also from their X-ray spectrum dominated by the soft component, in variance with the group $\beta$ (Mürset et al. 1996b) in which the harder component, likely due to the colliding winds, dominates.

Acknowledgements. This research has made use of NASA's Astrophysics Data System Abstract Service. I thank Dr. Hudec for reading the manuscript and for his comments. This research has made use of the electronic catalog of supersoft X-ray sources available at URL http://www.mpe.mpg.de/ jcg/sss/ssscat.html and maintained by J. Greiner. The support by the post-doctoral grant 205/00/P013 of the Grant Agency of the Czech Republic and the project ESA PRODEX INTEGRAL 14527 is acknowledged.

\section{References}

Aaronson, M., Liebert, J., \& Stocke, J. 1982, ApJ, 254, 507

Balman, S., Krautter, J., \& Oegelman, H. 1998, ApJ, 499, 395

Balman, S., \& Krautter, J. 2001, MNRAS, 326, 1441

Barlow, M. J., Brodie, J. P., Brunt, C. C., et al. 1981, MNRAS, 195, 61

Bearda, H., Hartmann, W., Ebisawa, K., et al. 2002, A\&A, 385, 511

Beuermann, K., Reinsch, K., Barwig, H., et al. 1995, A\&A, 294, L1

Bos, M., Retter, A., McCormick, J., \& Velthuis, F. 2001, IAU Circ. 7610

Celis, S. L. 1977, A\&AS, 29, 15

Celis, S. L. 1982, AJ, 87, 1791 
Chochol, D., Hric, L., Urban, Z., et al. 1993, A\&A, 277, 103

Chochol, D., Grygar, J., Pribulla, T., et al. 1997, A\&A, 318, 908

Cowley, A. P., Schmidtke, P. C., Crampton, D., \& Hutchings, J. B. 1990, ApJ, 350, 288

Cowley, A. P., Schmidtke, P. C., Crampton, D., et al. 1991, ApJ, 373, 228

Cowley, A. P., Schmidtke, P. C., Hutchings, J. B., et al. 1993, ApJ, 418, L63

Cowley, A. P., Schmidtke, P. C., McGrath, T. K., et al. 1997, PASP, 109,21

Crampton, D., Cowley, A. P., Hutchings, J. B., et al. 1987, ApJ, 321, 745

Crampton, D., Hutchings, J. B., Cowley, A. P., et al. 1996, ApJ, 456, 320

Crampton, D., Hutchings, J. B., Cowley, A. P., \& Schmidtke, P. C. 1997, ApJ, 489, 903

Della Valle, M., Pasquini, L., Daou, D., \& Williams, R. E. 2002, A\&A, 390,155

Deufel, B., Barwig, H., Simic, D., et al. 1999, A\&A, 343, 455

Diaz, M. P., \& Steiner, J. E. 1989, ApJ, 339, L41

Diaz, M. P., \& Steiner, J. E. 1995, AJ, 110, 1816

Diaz, M. P., Williams, R. E., Phillips, M. M., \& Hamuy, M. 1995, MNRAS, 277, 959

Ebisawa, K., Mukai, K., Kotani, T., et al. 2001, ApJ, 550, 1007

Eggen, O. J., Freeman, K. C., \& Sandage, A. 1968, ApJ, 154, L27

Eracleous, M., Halpern, J., \& Patterson, J. 1991, ApJ, 382, 290

Gális, R., Hric, L., Friedjung, M., \& Petrík, K. 1999, A\&A, 348, 533

Gould, A. 1995, ApJ, 452, 189

Greiner, J. 2000, NewA, 5, 137

Greiner, J., \& Wenzel, W. 1995, A\&A, 294, L5

Greiner, J., Bickert, K., Luthardt, R., et al. 1997, A\&A, 322, 576

Greiner, J., \& van Teeseling, A. 1998, A\&A, 339, L21

Greiner, J., Tovmassian, G. H., Di Stefano, R., et al. 1999, A\&A, 343, 183

Greiner, J., Orio, M., \& Schwarz, R. 2000, A\&A, 355, 1041

Gutierrez-Moreno, A., Moreno, H., \& Costa, E. 1999, PASP, 111, 571

Hachisu, I., Kato, M., Kato, T., \& Matsumoto, K. 2000, ApJ, 528, L97

Hack, M., \& la Dous, C. 1993, Cataclysmic variables and related objects, Monograph Series on Nonthermal Phenomena in Stellar Atmospheres-NASA SP-507, 15

Herbig, G. H., Preston, G. W., Smak, J., \& Paczynski, B. 1965, ApJ, 141,617

Hitner, W. A. 1956, ApJS, 2, 389

Hoard, D. W., Wallerstein, G., \& Willson, L. A. 1996, PASP, 108, 81

Hutchings, J. B., Cowley, A. P., Schmidtke, P. C., \& Crampton, D. 1995, AJ, 110, 2394

Iijima, T. 2002, A\&A, 387, 1013

Johnson, H. L. 1966, ARA\&A, 4, 193

Jordan, S., Murset, U., \& Werner, K. 1994, A\&A, 283, 475

Jordan, S., Schmutz, W., Wolff, B., et al. 1996, A\&A, 312, 897

Kahabka, P. 1998, A\&A, 331, 328

Kahabka, P., Parmar, A. N., \& Hartmann, H. W. 1999a, A\&A, 346, 453

Kahabka, P., Hartmann, H. W., Parmar, A. N., \& Negueruela, I. 1999b, A\&A, 347, L43

Kilmartin, P. M. 1999, IAU Circ. 7277

Knigge, Ch., King, A. R., \& Patterson, J. 2000, A\&A, 364, L75

Krautter, J., Beuermann, K., Leitherer, C., et al. 1984, A\&A, 137, 307

Kuduz, M., Reinsch, K., Beuermann, K., Kube, J. 2002, The Physics of Cataclysmic Variables and Related Objects, ed. B. T. Gansicke, K. Beuermann, \& K. Reinsch, ASP Conf. Proc., 261, 641 (Astronomical Society of the Pacific)

Matsumoto, K. 1996, PASJ, 48, 827
McGrath, T. K., Schmidtke, P. C., Cowley, A. P., et al. 2001, AJ, 122, 1578

Meade, M. R. 1999, AJ, 118, 1073

Mikolajewska, J., Kenyon, S. J., Mikolajewski, M., et al. 1995, AJ, 109,1289

Morgan, D. H. 1992, MNRAS, 258, 639

Motch, C., Hasinger, G., \& Pietsch, W. 1994, A\&A, 284, 827

Motch, C. 1996, Supersoft X-Ray Sources, Lecture Notes Phys., 472, 83

Motch, C., Bearda, H., \& Neiner, C. 2002, A\&A, 393, 913

Mumford, G. S. 1971, ApJ, 165, 369

Munari, U. 1991, IBVS, 3605, 1

Munari, U., Iudin, B. F., Taranova, O. G., et al. 1992, A\&AS, 93, 383

Munari, U., \& Buson, L. M. 1994, A\&A, 287, 87

Munari, U., Zwitter, T., \& Bragaglia, A. 1997, A\&AS, 122, 495

Mürset, U., Nussbaumer, H., Schmid, H. M., \& Vogel, M. 1991, A\&A, 248,458

Mürset, U., Jordan, S., \& Wolff, B. 1996a, Supersoft X-Ray Sources, Lecture Notes Phys., 472, 251

Mürset, U., Schild, H., \& Vogel, M. 1996b, A\&A, 307, 516

Oegelman, H., Orio, M., Krautter, J., \& Starrfield, S. 1993, Nature, 361,331

Oliveira, A. S., \& Steiner, J. E. 2002, The Physics of Cataclysmic Variables and Related Objects, ed. B. T. Gansicke, K. Beuermann, \& K. Reinsch, ASP Conf. Proc., 261, 649 (Astronomical Society of the Pacific)

Orio, M., della Valle, M., Massone, G., \& Oegelman, H. 1997, A\&A, 325, L1

Orio, M., Parmar, A. N., Capalbi, M., Piro, L., \& Mineo, T. 1999, IAU Circ. 7325

Paczynski, B. 1977, ApJ, 216, 822

Pakull, M. W., Moch, C., Bianchi, L., et al. 1993, A\&A, 278, L39

Patterson, J., Thorstensen, J. R., Fried, R., et al. 2001, PASP, 113, 72

Popham, R., \& Di Stefano, R. 1996, Supersoft X-Ray Sources, Lecture Notes Phys., 472, 99

Rauch, T. 1996, Supersoft X-Ray Sources, Lecture Notes Phys., 472, 139

Reinsch, K., van Teeseling, A., Beuermann, K., \& Thomas, H.-C. 1996a, Supersoft X-Ray Sources, Lecture Notes Phys., 472, 173

Reinsch, K., van Teeseling, A., Beuermann, K., \& Thomas, H.-C. 1996b, Proc. Rontgenstrahlung from the Universe, ed. Zimmermann, H. U., Trumper, J., \& Yorke, H., MPE Rep., 263,183

Reinsch, K., van Teeseling, A., King, A. R., \& Beuermann, K. 2000, A\&A, 354, L37

Schaefer, B. E., \& Ringwald, F. A. 1995, ApJ, 447, L45

Schmid, H. M., \& Nussbaumer, H. 1993, A\&A, 268, 159

Schmid, H. M., Dumm, T., Murset, U., et al. 1998, A\&A, 329, 986

Schmidtke, P. C., McGrath, T. K., Cowley, A. P., \& Frattare, L. M. 1993, PASP, 105, 863

Schmidtke, P. C., \& Cowley, A. P. 1995, IAU Circ., 6278

Schmidtke, P. C., \& Cowley, A. P. 1996, AJ, 112, 167

Schmidtke, P. C., Cowley, A. P., McGrath, T. K., et al. 1996, AJ, 111, 788

Schmidtke, P. C., Cowley, A. P., Taylor, V. A., et al. 2000, AJ, 120, 935

Schmidtke, P. C., \& Cowley, A. P. 2001, AJ, 122, 1569

Sekiguchi, K., Feast, M. W., Whitelock, P. A., et al. 1988, MNRAS, 234, 281

Shanley, L., Ogelman, H., Gallagher, J. S., et al. 1995, ApJ, 438, L95

Skillman, D. R., Harvey, D., Patterson, J., \& Vanmunster, T. 1997, PASP, 109, 114

Smak, J., Belczynski, K., \& Zola, S., 2001, Acta Astron., 51, 117 
Smale, A. P., Corbet, R. H. D., Charles, P. A., et al. 1988, MNRAS, 233, 51

Steiner, J. E., \& Diaz, M. P. 1998, PASP, 110, 276

Stetson, P. B. 1979, AJ, 84, 1149

Szkody, P. 1994, AJ, 108, 639

Šimon, V., Shugarov, S., \& Marsakova, V. I. 2001, A\&A, 366, 100

Šimon, V. 2002, Classical Nova Explosions: International Conference on Classical Nova Explosions, Sitges, Spain, ed. M. Hernanz, \& J. José, AIP Conf. Proc., 637, 338

Theuns, T., \& Jorissen, A. 1993, MNRAS, 265, 946

Tomov, N. A., Tomova, M. T., \& Ivanova, A. 2000, A\&A, 364, 557

van den Bergh, S. 1989, A\&ARv, 1, 111

van den Heuvel, E. P. J., Bhattacharya, D., Nomoto, K., \& Rappaport, S. A. 1992, A\&A, 262, 97

van Teeseling, A., Reinsch, K., Beuermann, K., et al. 1996, Supersoft X-Ray Sources, Lecture Notes Phys., 472, 115 van Teeseling, A., Reinsch, K., Hessman, F. V., \& Beuermann, K. 1997, A\&A, 323, L41

van Teeseling, A., Reinsch, K., Pakull, M. W., \& Beuermann, K. 1998, A\&A, 338, 947

van Teeseling, A., \& King, A. R. 1998, A\&A, 338, 957

Viotti, R., Gonzales-Riestra, R., Montagni, F., et al. 1996, Supersoft X-Ray Sources, Lecture Notes Phys., 472, 259

Warner, B. 1995, Cataclysmic Variable Stars (Cambridge: Cambridge Univ. Press)

Whitelock, P. A. 1987, PASP, 99, 573

Whitelock, P. A. 1988, IAU Coll., 103, 47

Will, T., \& Barwig, H. 1996, Supersoft X-Ray Sources, Lecture Notes Phys., 472, 99

Willson, L. A., Garnavich, P., \& Mattei, J. A. 1981, IBVS 1961

Woodward, C. E., Gehrz, R. D., Jones, T. J., et al. 1997, ApJ, 477, 817 


\section{Online Material}


Table 2. Summary of the literature used for the determination of the parameters of the SSXBs, listed in Tables 1 and 3.

QR And/RX J0019.8+2156: indices:Matsumoto (1996), Deufel et al. (1999), Beuermann et al. (1995); $d, E(B-V)$ and $L_{\mathrm{bol}}$ : Beuermann et al. (1995); $P_{\mathrm{orb}}$ : Will \& Barwig (1996)

MR Vel/RX J0925.7-4758: indices:Schmidtke et al. (2000),Motch et al. (1994); $P_{\text {orb }}$ : Schmidtke \& Cowley (2001); $d$ and $E(B-V)$ : Motch (1996); $L_{\mathrm{bol}}$ : Bearda et al. (2002)

V Sge: indices:Šimon et al. (2001); $d$ and $E(B-V)$ : Smak et al. (2001); $P_{\mathrm{orb}}$ : Herbig et al. (1965); $L_{\mathrm{bol}}$ : Greiner \& van Teeseling (1998)

V 617 Sgr: indices, $P_{\text {orb }}, E(B-V)$ : Steiner \& Diaz (1998)

WX Cen: indices: Eggen et al. (1968), Mumford (1971); $P_{\text {orb }}, E(B-V)$ : Diaz \& Steiner (1995)

HD 45166: indices, $E(B-V)$ : Meade (1999); $P_{\text {orb }}$ : Oliveira \& Steiner (2002); $d$ : Hitner (1956)

V 1974 Cyg/Nova Cyg 1992: indices:Chochol et al. (1993); $d$ and $E(B-V)$ : Chochol et al. (1997); X-ray activity and $L_{\text {bol }}$ : Balman et al. (1998); $P_{\text {orb }}$ : Skillman et al. (1997)

GQ Mus/Nova Mus 1983: indexes:Diaz \& Steiner (1989), Diaz et al. (1995); $d$ and $E(B-V)$ : Krautter et al. (1984); $P_{\text {orb }}$ : Diaz \& Steiner (1989); X-ray activity: Shanley et al. (1995); $L_{\text {bol }}$ : Oegelman et al. (1993), Balman \& Krautter (2001) V 382 Vel: indices: Kilmartin (1999); $d, E(B-V)$ : Della Valle et al. (2002); $P_{\text {orb }}$ : Bos et al. (2001); X-ray activity and $L_{\text {bol }}$ : Orio et al. (1999)

U Sco: indices:Barlow et al. (1981), Sekiguchi et al. (1988); $P_{\text {orb }}$ : Schaefer \& Ringwald (1995); $d$ : Hachisu et al. (2000); $L_{\text {bol }}$ : Kahabka et al. (1999b)

RR Tel: indices: Munari et al. (1992); $d$ : Whitelock (1988); $E(B-V)$ : Mürset et al. (1991); $L_{\mathrm{bol}}$ : Jordan et al. (1994)

AG Dra: indices: Greiner et al. (1997), Viotti et al. (1996); $L_{\mathrm{bol}}$ : Greiner et al. (1997); $E(B-V)$ : Mikolajewska et al. (1995); $d$ : Tomov et al. (2000); $P_{\text {orb }}$ : Gális et al. (1999)

CD-43 14304: indices: Munari et al. (1992), Gutierrez-Moreno et al. (1999); $E(B-V)$ : Schmid \& Nussbaumer (1993); $d$ : Gutierrez-Moreno et al. (1999); $P_{\text {orb }}$ : Schmid et al. (1998)

R Aqr: indices: Celis S $(1977,1982) ; E(B-V), d$ : Whitelock (1987); $P_{\text {orb }}$ : Willson et al. (1981)

V 751 Cyg: indices:Munari et al. (1997), Greiner et al. (1999); $d, E(B-V)$ and $L_{\mathrm{bol}}$ : Greiner et al. (1999); $P_{\mathrm{orb}}$ : Patterson et al. (2001)

RX J0513-69: indices:Steiner \& Diaz (1998), Pakull et al. (1993), Cowley et al. (1993); $P_{\text {orb }}$ Crampton et al. (1996); $L_{\text {bol }}$ : Reinsch et al. (1996a)

RX J0439.8-6809: indices: Schmidtke \& Cowley (1995), Reinsch et al. (1996b), van Teeseling et al. (1996), Schmidtke \& Cowley (1996); $P_{\text {orb }}$ : Schmidtke \& Cowley (1996), van Teeseling et al. (1997); $L_{\mathrm{bol}}$ : van Teeseling et al. (1996)

RX J0537.7-7034: indices and $L_{\mathrm{bol}}$ :Orio et al. (1997), Greiner et al. (2000); $P_{\mathrm{orb}}$ : Greiner et al. (2000)

CAL 83: indices:Steiner \& Diaz (1998), Crampton et al. (1987), Smale et al. (1988); $P_{\text {orb }}$ :Smale et al. (1988); $L_{\text {bol }}$ : Kahabka (1998)

CAL 87: indices: Steiner \& Diaz (1998), Cowley et al. (1990), Cowley et al. (1991); $P_{\text {orb }}$ Schmidtke et al. (1993); $E(B-V)$ : Hutchings et al. (1995); $L_{\mathrm{bol}}$ : Ebisawa et al. (2001)

1E 0035.4-7230: indices, $P_{\text {orb }}, E(B-V), d$ : van Teeseling et al. (1998); indices: Crampton et al. (1997), Schmidtke et al. (1996); $L_{\text {bol }}$ : Kahabka et al. (1999a)

RX J0048.4-7332: indices: Cowley et al. (1997); $d, E(B-V), L_{\mathrm{bol}}$ : Jordan et al. (1996); outburst: Morgan (1992)

Ln358: indices, $E(B-V), d$ : Mürset et al. (1996b)

Draco C-1: indices: Aaronson et al. (1982), Munari (1991); $d, E(B-V)$ : Stetson (1979); X-ray activity: Mürset et al. (1996a) 
V. Šimon: Colors of the supersoft X-ray sources, Online Material $p 3$

Table 3. The colors of the objects with the soft X-ray emission from Table 1. Both the observed and dereddened colors are given. The columns labeled as $V$ and $M_{V 0}$, respectively, give the apparent and absolute magnitude to which the color refers. When available, the appropriate Julian Date (JD-2 400000 ) or a year are included. The time interval after the outburst maximum $t-T_{\max }$ (days) is listed for the classical and recurrent novae.

\begin{tabular}{|c|c|c|c|c|c|c|c|c|c|c|c|c|}
\hline object & $V$ & $M_{V 0}$ & $U-B$ & $(U-B)_{0}$ & $B-V$ & $(B-V)_{0}$ & $V-R$ & $(V-R)_{0}$ & $R-I$ & $(R-I)_{0}$ & JD & $t-T_{\mathrm{max}}$ \\
\hline \multicolumn{13}{|l|}{ Galaxy } \\
\hline \multirow[t]{7}{*}{ QR And } & 12.16 & 0.34 & -0.66 & -0.73 & 0.14 & 0.04 & 0.12 & 0.07 & 0.17 & 0.11 & $\sim 48884$ & \\
\hline & 12.65 & 0.83 & -0.70 & -0.77 & 0.11 & 0.01 & 0.10 & 0.05 & 0.19 & 0.13 & $\sim 48884$ & \\
\hline & 12.37 & 0.55 & -1.09 & -1.16 & 0.15 & 0.05 & 0.18 & 0.13 & & & $\sim 48893$ & \\
\hline & 12.69 & 0.87 & -1.11 & -1.18 & 0.13 & 0.03 & 0.20 & 0.15 & & & $\sim 48893$ & \\
\hline & 12.6 & 0.78 & & & 0.01 & -0.09 & 0.09 & 0.04 & 0.10 & 0.04 & 49993 & \\
\hline & 12.33 & 0.51 & -0.92 & -0.99 & -0.04 & -0.14 & & & & & 50369 & \\
\hline & 12.65 & 0.83 & -0.92 & -0.99 & 0.06 & -0.04 & & & & & 50369 & \\
\hline \multirow[t]{2}{*}{$\overline{\mathrm{MR} \text { Vel }}$} & 17.2 & 0.12 & & & 2.01 & 0.01 & 1.26 & 0.05 & 1.30 & -0.05 & $\sim 48974$ & \\
\hline & 17.2 & 0.12 & & & 1.9 & -0.1 & 1.27 & 0.06 & & & 50859 & \\
\hline \multirow[t]{12}{*}{ V Sge } & 12.4 & -1.63 & -0.89 & -1.13 & 0.04 & -0.29 & & & & & mean & \\
\hline & 12.2 & -1.83 & -0.90 & -1.13 & 0.04 & -0.29 & & & & & colors & \\
\hline & 12.0 & -2.03 & -0.90 & -1.14 & 0.04 & -0.29 & & & & & in the & \\
\hline & 11.8 & -2.23 & -0.90 & -1.14 & 0.04 & -0.29 & & & & & years & \\
\hline & 11.6 & -2.43 & -0.90 & -1.14 & 0.03 & -0.29 & & & & & 1995- & \\
\hline & 11.4 & -2.63 & -0.89 & -1.13 & 0.03 & -0.30 & & & & & 1997 & \\
\hline & 11.2 & -2.83 & -0.88 & -1.12 & 0.01 & -0.32 & & & & & & \\
\hline & 11.0 & -3.03 & -0.88 & -1.12 & -0.01 & -0.34 & & & & & & \\
\hline & 10.8 & -3.23 & -0.88 & -1.12 & -0.04 & -0.37 & & & & & & \\
\hline & 10.6 & -3.43 & -0.88 & -1.12 & -0.06 & -0.39 & & & & & & \\
\hline & 10.4 & -3.63 & -0.87 & -1.11 & -0.09 & -0.42 & & & & & & \\
\hline & 10.3 & -3.73 & -0.87 & -1.11 & -0.10 & -0.43 & & & & & & \\
\hline V617 Sgr & 14.8 & & -0.87 & -1.01 & -0.04 & -0.24 & & & & & 46966 & \\
\hline \multirow[t]{3}{*}{ WX Cen } & 13.7 & 1.73 & -0.72 & -1.02 & 0.4 & 0.0 & & & & & $\sim 40059$ & \\
\hline & 13.51 & 1.54 & -0.56 & -0.86 & 0.50 & 0.10 & & & & & 40265.78 & \\
\hline & 13.53 & 1.56 & -0.61 & -0.91 & 0.50 & 0.10 & & & & & 40267.79 & \\
\hline HD 45166 & 9.88 & -1.08 & -0.76 & -0.87 & -0.07 & -0.22 & & & & & & \\
\hline \multirow[t]{6}{*}{ V1974 Cyg } & 9.43 & -2.80 & -1.12 & -1.35 & -0.07 & -0.39 & & & & & 48883.35 & 209.6 \\
\hline & 9.47 & -2.76 & -1.08 & -1.31 & -0.11 & -0.43 & & & & & 48890.39 & 216.7 \\
\hline & 9.71 & -2.52 & -1.20 & -1.43 & -0.21 & -0.53 & & & & & 48904.41 & 230.7 \\
\hline & 9.53 & -2.70 & -1.13 & -1.36 & -0.02 & -0.34 & & & & & 48909.39 & 235.7 \\
\hline & 9.53 & -2.70 & -1.12 & -1.35 & -0.01 & -0.33 & & & & & 48910.36 & 236.7 \\
\hline & 9.91 & -2.32 & -1.70 & -1.93 & -0.01 & -0.33 & & & & & 48936.27 & 263.7 \\
\hline \multirow[t]{2}{*}{ GQ Mus } & 17.5 & 2.70 & -0.75 & -1.07 & 0.15 & -0.30 & & & & & 47242 & \\
\hline & 18.0 & 3.20 & -0.7 & -1.02 & 0.31 & -0.14 & & & & & 49391 & \\
\hline V382 Vel & 9.05 & -3.07 & -1.24 & -1.38 & -0.55 & -0.75 & 0.60 & 0.51 & -0.54 & -0.68 & 51464.17 & 142.7 \\
\hline \multirow[t]{5}{*}{ U Sco } & 11.92 & -2.59 & -0.60 & -0.74 & -0.25 & -0.45 & 0.39 & 0.30 & 0.15 & 0.01 & 44054.24 & 5.7 \\
\hline & 12.01 & -2.50 & -0.63 & -0.77 & -0.15 & -0.35 & 0.37 & 0.28 & 0.23 & 0.09 & 44055.31 & 6.8 \\
\hline & 12.77 & -1.74 & -0.64 & -0.78 & -0.28 & -0.48 & 0.41 & 0.32 & 0.24 & 0.10 & 44056.25 & 7.7 \\
\hline & 13.33 & -1.18 & -0.73 & -0.87 & -0.22 & -0.42 & 0.34 & 0.25 & 0.24 & 0.10 & 44057.27 & 8.7 \\
\hline & 12.47 & -2.04 & -0.58 & -0.72 & -0.30 & -0.50 & 0.47 & 0.38 & 0.18 & 0.04 & 46934.53 & $3-7$ \\
\hline \multirow[t]{2}{*}{ RR Tel } & 10.84 & -1.54 & -1.36 & -1.43 & 0.42 & 0.32 & 0.53 & 0.48 & & & 48066 & \\
\hline & 10.84 & -1.54 & -1.41 & -1.48 & 0.40 & 0.30 & 0.52 & 0.47 & & & 48067 & \\
\hline \multirow[t]{12}{*}{ AG Dra } & 9.76 & -1.58 & 0.62 & 0.58 & 1.39 & 1.33 & & & & & 48127.38 & \\
\hline & 9.85 & -1.49 & 0.40 & 0.36 & 1.41 & 1.35 & & & & & 48179.35 & \\
\hline & 9.83 & -1.51 & 0.17 & 0.13 & 1.37 & 1.31 & & & & & 48274.63 & \\
\hline & 9.76 & -1.58 & 0.22 & 0.18 & 1.34 & 1.28 & & & & & 48329.62 & \\
\hline & 9.76 & -1.58 & 0.23 & 0.19 & 1.35 & 1.29 & & & & & 48332.58 & \\
\hline & 9.69 & -1.65 & 0.30 & 0.26 & 1.37 & 1.31 & & & & & 48353.53 & \\
\hline & 9.70 & -1.64 & 0.24 & 0.20 & 1.37 & 1.31 & & & & & 48359.50 & \\
\hline & 9.72 & -1.62 & 0.26 & 0.22 & 1.35 & 1.29 & & & & & 48362.53 & \\
\hline & 9.73 & -1.61 & 0.20 & 0.16 & 1.35 & 1.29 & & & & & 48409.44 & \\
\hline & 9.71 & -1.63 & 0.34 & 0.30 & 1.38 & 1.32 & & & & & 48440.47 & \\
\hline & 9.77 & -1.57 & 0.52 & 0.48 & 1.38 & 1.32 & & & & & 48494.39 & \\
\hline & 9.74 & -1.60 & 0.53 & 0.49 & 1.37 & 1.31 & & & & & 48500.42 & \\
\hline
\end{tabular}


V. Šimon: Colors of the supersoft X-ray sources, Online Material p 4

Table 3. continued.

\begin{tabular}{|c|c|c|c|c|c|c|c|c|c|c|c|c|}
\hline object & $V$ & $M_{V 0}$ & $U-B$ & $(U-B)_{0}$ & $B-V$ & $(B-V)_{0}$ & $V-R$ & $(V-R)_{0}$ & $R-I$ & $(R-I)_{0}$ & JD & $t-T_{\max }$ \\
\hline \multirow[t]{33}{*}{ AG Dra } & 9.71 & -1.63 & 0.46 & 0.42 & 1.37 & 1.31 & & & & & 48681.47 & \\
\hline & 9.73 & -1.61 & 0.51 & 0.47 & 1.35 & 1.29 & & & & & 48691.61 & \\
\hline & 9.72 & -1.62 & 0.31 & 0.27 & 1.30 & 1.24 & & & & & 48747.46 & \\
\hline & 9.75 & -1.59 & 0.27 & 0.23 & 1.33 & 1.27 & & & & & 48801.43 & \\
\hline & 9.74 & -1.60 & 0.25 & 0.21 & 1.34 & 1.28 & & & & & 48803.44 & \\
\hline & 9.85 & -1.49 & 0.22 & 0.18 & 1.34 & 1.28 & & & & & 48830.40 & \\
\hline & 9.83 & -1.51 & 0.13 & 0.09 & 1.35 & 1.29 & & & & & 48843.37 & \\
\hline & 9.41 & -1.93 & -0.51 & -0.55 & 0.99 & 0.93 & & & & & 49213.37 & \\
\hline & 9.48 & -1.86 & -0.11 & -0.15 & 1.05 & 0.99 & & & & & 49214.43 & \\
\hline & 9.54 & -1.80 & -0.34 & -0.39 & 1.08 & 1.02 & & & & & 49215.39 & \\
\hline & 9.49 & -1.85 & -0.42 & -0.46 & 1.08 & 1.02 & & & & & 49217.35 & \\
\hline & 9.63 & -1.71 & -0.27 & -0.31 & 1.20 & 1.14 & & & & & 49249.33 & \\
\hline & 9.64 & -1.70 & -0.28 & -0.32 & 1.18 & 1.12 & & & & & 49250.35 & \\
\hline & 8.89 & -2.45 & -0.96 & -1.00 & 0.67 & 0.61 & & & & & 49534.48 & \\
\hline & 8.77 & -2.57 & -0.91 & -0.95 & 0.57 & 0.51 & & & & & 49535.43 & \\
\hline & 8.67 & -2.67 & -0.90 & -0.94 & 0.55 & 0.49 & & & & & 49537.44 & \\
\hline & 8.46 & -2.88 & -0.92 & -0.96 & 0.50 & 0.44 & & & & & 49541.46 & \\
\hline & 8.55 & -2.79 & -0.87 & -0.91 & 0.47 & 0.41 & & & & & 49545.42 & \\
\hline & 8.61 & -2.73 & -0.79 & -0.83 & 0.48 & 0.42 & & & & & 49568.41 & \\
\hline & 8.62 & -2.72 & -0.83 & -0.87 & 0.49 & 0.43 & & & & & 49569.41 & \\
\hline & 8.68 & -2.66 & & & 0.65 & 0.59 & 0.55 & 0.52 & 0.44 & 0.40 & 49586 & \\
\hline & 8.85 & -2.49 & -0.78 & -0.82 & 0.65 & 0.59 & & & & & 49599.36 & \\
\hline & 9.06 & -2.28 & -0.71 & -0.75 & 0.72 & 0.66 & & & & & 49623.41 & \\
\hline & 9.07 & -2.27 & -0.71 & -0.75 & 0.73 & 0.67 & & & & & 49625.43 & \\
\hline & 9.09 & -2.25 & & & 0.89 & 0.83 & 0.66 & 0.63 & 0.60 & 0.56 & 49633 & \\
\hline & 9.17 & -2.17 & -0.73 & -0.77 & 0.75 & 0.69 & & & & & 49638.37 & \\
\hline & 9.15 & -2.19 & -0.71 & -0.75 & 0.81 & 0.75 & & & & & 49639.37 & \\
\hline & 9.19 & -2.15 & -0.77 & -0.81 & 0.78 & 0.72 & & & & & 49644.44 & \\
\hline & 9.14 & -2.20 & & & 0.76 & 0.70 & 0.69 & 0.66 & 0.57 & 0.53 & 49960 & \\
\hline & 9.46 & -1.88 & & & 0.97 & 0.91 & 0.81 & 0.78 & 0.55 & 0.51 & 50016 & \\
\hline & 9.59 & -1.75 & & & 1.08 & 1.02 & 0.79 & 0.76 & 0.63 & 0.59 & 50064 & \\
\hline & 9.57 & -1.77 & & & 1.16 & 1.10 & 0.76 & 0.73 & 0.68 & 0.64 & 50112 & \\
\hline & 9.55 & -1.79 & & & 1.07 & 1.01 & 0.74 & 0.71 & 0.68 & 0.64 & 50150 & \\
\hline \multirow{15}{*}{ R Aqr } & 11.69 & 4.70 & -0.62 & -0.62 & 1.04 & 1.04 & 4.36 & 4.36 & 3.03 & 3.03 & 40891.53 & \\
\hline & 11.69 & 4.70 & -0.58 & -0.58 & 1.04 & 1.04 & 4.44 & 4.44 & 3.03 & 3.03 & 40893.58 & \\
\hline & 11.56 & 4.57 & -0.60 & -0.60 & 1.01 & 1.01 & 4.41 & 4.41 & 3.06 & 3.06 & 40894.56 & \\
\hline & 11.99 & 5.00 & -0.53 & -0.53 & 0.87 & 0.87 & 4.73 & 4.73 & 3.21 & 3.21 & 40922.61 & \\
\hline & 11.88 & 4.89 & -0.66 & -0.66 & 0.95 & 0.95 & 4.70 & 4.70 & 3.21 & 3.21 & 40947.56 & \\
\hline & 11.51 & 4.52 & -0.52 & -0.52 & 1.10 & 1.10 & 4.74 & 4.74 & 3.16 & 3.16 & 40974.57 & \\
\hline & 7.57 & 0.58 & -0.07 & -0.07 & 1.72 & 1.72 & & & & & 41655.67 & \\
\hline & 8.64 & 1.65 & -0.43 & -0.43 & 1.75 & 1.75 & & & & & 41688.59 & \\
\hline & 11.41 & 4.42 & -0.22 & -0.22 & 1.24 & 1.24 & & & & & 41834.34 & \\
\hline & 11.05 & 4.06 & -0.29 & -0.29 & 1.50 & 1.50 & & & & & 41896.79 & \\
\hline & 10.57 & 3.58 & -0.12 & -0.12 & 1.29 & 1.29 & & & & & 41925.09 & \\
\hline & 8.71 & 1.72 & 0.38 & 0.38 & 1.43 & 1.43 & & & & & 41979.59 & \\
\hline & 7.64 & 0.65 & 0.53 & 0.53 & 1.47 & 1.47 & & & & & 42009.09 & \\
\hline & 8.11 & 1.12 & 0.11 & 0.11 & 1.48 & 1.48 & & & & & 42045.89 & \\
\hline & 8.80 & 1.81 & -0.06 & -0.06 & 1.60 & 1.60 & & & & & 42068.55 & \\
\hline \multirow[t]{4}{*}{ CD-43 ${ }^{\circ} 14304$} & 12.53 & 1.05 & & & 1.17 & 0.97 & & & & & yr 1987 & \\
\hline & 11.55 & 0.07 & 0.39 & 0.24 & 1.57 & 1.37 & 0.94 & 0.82 & 0.81 & 0.67 & 48066 & \\
\hline & 11.55 & 0.07 & 0.36 & 0.21 & 1.59 & 1.39 & 0.93 & 0.81 & 0.81 & 0.67 & 48067 & \\
\hline & 12.14 & 0.66 & & & 1.05 & 0.85 & & & & & yr 1991 & \\
\hline \multirow{2}{*}{ V751 Cyg } & 14.3 & 5.04 & & & 0.36 & 0.11 & 0.20 & 0.09 & & & 49630.4 & \\
\hline & 16.08 & 6.82 & -0.64 & -0.82 & 0.09 & -0.16 & 0.50 & 0.39 & 0.22 & 0.05 & 50786 & \\
\hline \multicolumn{13}{|l|}{$\overline{\text { LMC }}$} \\
\hline \multirow[t]{4}{*}{ J0513 } & 16.7 & -1.98 & & & -0.14 & -0.24 & & & & & $\sim 48735$ & \\
\hline & 16.7 & -1.98 & & & -0.11 & -0.21 & & & & & Dec. 92 , Mar. 93 & \\
\hline & 16.6 & -2.08 & & & 0.06 & -0.04 & 0.08 & 0.03 & & & 49630 & \\
\hline & 17.55 & -1.13 & & & 0.14 & 0.04 & 0.20 & 0.15 & & & 49698 & \\
\hline
\end{tabular}


V. Šimon: Colors of the supersoft X-ray sources, Online Material p 5

Table 3. continued.

\begin{tabular}{|c|c|c|c|c|c|c|c|c|c|c|c|c|}
\hline object & $V$ & $M_{V 0}$ & $U-B$ & $(U-B)_{0}$ & $B-V$ & $(B-V)_{0}$ & $V-R$ & $(V-R)_{0}$ & $R-I$ & $(R-I)_{0}$ & JD & $t-T_{\max }$ \\
\hline \multirow[t]{4}{*}{ J0439 } & 21.75 & 3.07 & & & -0.35 & -0.45 & & & & & $\sim 49700$ & \\
\hline & 21.74 & 3.06 & -1.1 & -1.17 & -0.24 & -0.34 & & & & & $\sim 50000$ & \\
\hline & 21.6 & 2.92 & -1.25 & -1.32 & -0.29 & -0.39 & & & & & 50047 & \\
\hline & 21.8 & & -1.22 & -1.29 & -0.36 & -0.46 & & & & & Nov. 95 & \\
\hline \multirow[t]{2}{*}{ J0537 } & 19.66 & 0.98 & -0.69 & -0.76 & -0.03 & -0.13 & & & & & 49721.65 & \\
\hline & 19.7 & 1.02 & & & -0.05 & -0.15 & & & & & 50833 & \\
\hline \multirow[t]{3}{*}{ CAL 83} & 17.3 & -1.38 & -1.1 & -1.17 & 0.10 & 0.00 & & & & & & \\
\hline & 17.28 & -1.40 & & & -0.02 & -0.12 & & & & & $\sim 46382$ & \\
\hline & 17.28 & -1.40 & & & -0.01 & -0.11 & & & & & $\sim 50393$ & \\
\hline CAL 87 & 18.90 & 0.38 & -0.74 & -0.78 & 0.14 & 0.09 & 0.12 & 0.10 & 0.08 & 0.05 & $\sim 47861$ & \\
\hline \multicolumn{13}{|l|}{ SMC } \\
\hline \multirow[t]{3}{*}{ 1E0035 } & 20.2 & 1.21 & -1.06 & -1.10 & -0.15 & -0.21 & & & & & Dec. 93, Nov 94 & \\
\hline & 20.3 & 1.31 & -1.3 & -1.34 & -0.1 & -0.16 & 0.0 & -0.03 & & & 50435 & \\
\hline & 20.4 & 1.41 & -1.13 & -1.17 & -0.13 & -0.19 & & & & & Nov. 95 , Nov. 96 & \\
\hline J0048 & 15.0 & -3.95 & 0.2 & 0.16 & 1.5 & 1.45 & & & & & yr 1993 & \\
\hline Ln358 & 15.2 & -3.85 & & & 1.6 & 1.52 & 1.2 & 1.15 & 1.0 & 0.95 & 49314 & \\
\hline \multicolumn{13}{|c|}{ Draco dwarf galaxy C } \\
\hline \multirow[t]{3}{*}{ Draco C-1 } & 17.00 & -2.50 & & & 1.43 & 1.40 & 0.89 & 0.87 & 0.44 & 0.42 & 44703 & \\
\hline & 17.14 & -2.36 & & & 1.51 & 1.48 & & & & & 44731 & \\
\hline & 16.8 & -2.70 & & & 2.1 & 2.07 & & & & & 48093.49 & \\
\hline
\end{tabular}

